\title{
Revolutionaries and spies on trees and unicyclic graphs
}

\author{
Daniel W. Cranston, Clifford D. Smyth \\ and Douglas B. West*
}

\begin{abstract}
A team of $r$ revolutionaries and a team of $s$ spies play a game on a graph $G$. Initially, revolutionaries and then spies take positions at vertices. In each subsequent round, each revolutionary may move to an adjacent vertex or not move, and then each spy has the same option. The revolutionaries want to hold an unguarded meeting, meaning $m$ revolutionaries at some vertex having no spy at the end of a round. To prevent this forever, trivially at least $\min \{|V(G)|,\lfloor r / m\rfloor\}$ spies are needed. When $G$ is a tree, this many spies suffices. When $G$ is a unicyclic graph, $\min \{|V(G)|,\lceil r / m\rceil\}$ spies suffice, and we characterize those unicyclic graphs where $\lfloor r / m\rfloor+1$ spies are needed.
\end{abstract}

AMS 2000 SUBJECT Classifications: 05C57, 05C05, 05C35.

KEYWORDS AND PHRASES: Pursuit game, revolutionaries and spies, cops and robber, unicyclic graph.

\section{Introduction}

Many pursuit games have been studied on graphs. We study such a game that can be interpreted as modeling a problem of network security. One team consists of $r$ revolutionaries; the other consists of $s$ spies. The revolutionaries want to arrange a one-time meeting of $m$ revolutionaries free of oversight by spies. Initially, the revolutionaries take positions at vertices, and then the spies do the same. In each subsequent round, each revolutionary may move to an adjacent vertex or not move, and then each spy has the same option. Everyone knows where everyone else is.

The revolutionaries win if at the end of a round there is an unguarded meeting, where a meeting is a set of (at least) $m$ revolutionaries on one vertex, and a meeting is unguarded if there is no spy at that vertex. The spies win if they can prevent this forever. Let $\operatorname{RS}(G, m, r, s)$ denote this game played on

\footnotetext{
*Partially supported by NSA grant H98230-10-1-0363.
} 
the graph $G$ by $s$ spies and $r$ revolutionaries seeking an unguarded meeting of size $m$.

The revolutionaries can form $\min \{|V(G)|,\lfloor r / m\rfloor\}$ meetings initially; if $s$ is smaller than this, then the spies immediately lose. On the other hand, the spies win if $s \geq r-m+1$; they follow $r-m+1$ distinct revolutionaries, and the other $m-1$ revolutionaries cannot form a meeting. For fixed $G, r, m$ we study the minimum $s$ such that the spies win $\operatorname{RS}(G, m, r, s)$; let $\sigma(G, m, r)$ denote this threshold. The trivial bounds are

$$
\min \{|V(G)|,\lfloor r / m\rfloor\} \leq \sigma(G, m, r) \leq \min \{|V(G)|, r-m+1\}
$$

The game was invented by Jozef Beck in the mid-1990s. Shortly thereafter, Smyth proved that the trivial lower bound on $\sigma(G, m, r)$ is sufficient when $G$ is a tree. This was not published; we include a proof here and use the result in solving the game for unicyclic graphs. Howard and Smyth [2] studied the game when $G$ is the infinite 2-dimensional integer grid with onestep horizontal, vertical, and diagonal edges. They observed that the spy wins $\operatorname{RS}(G, m, 2 m-1,1)$ (the spy stays at the median position), and hence $\sigma(G, m, r) \leq r-2 m+2$ for general $r$ and $m$. For $m=2$, they proved that $6\lfloor r / 8\rfloor \leq \sigma(G, 2, r) \leq r-2$; they conjectured that the upper bound is the correct answer.

In this paper, we determine $\sigma(G, m, r)$ for all trees and unicyclic graphs. For a graph $G$ with $r / m<|V(G)|$, we show that $\sigma(G, m, r) \leq\lceil r / m\rceil$ if $G$ has at most one cycle, by summing over components. When $G$ is a tree, $\lfloor r / m\rfloor$ spies suffice, and $\lceil r / m\rceil$ suffice when $G$ is connected and unicyclic. Our final result is that if $G$ is unicyclic with a cycle of length $\ell$ and $t$ vertices not on the cycle (connected or not), and $m \nmid r$, then $\sigma(G, m, r)=\lfloor r / m\rfloor$ if and only if $\ell \leq \max \{\lfloor r / m\rfloor-t+2,3\}$.

Butterfield, Cranston, Puleo, West, and Zamani [1] study $\sigma(G, m, r)$ on a variety of graphs. With $r / m<|V(G)|$, they show that $\sigma(G, m, r)=\lfloor r / m\rfloor$ when $G$ has a rooted spanning tree $T$ such that every edge of $G$ not in $T$ joins vertices having the same parent in $T$; this includes graphs with a dominating vertex and interval graph. For every graph $G$, they prove $\sigma(G, m, r) \leq \gamma(G)\lfloor r / m\rfloor$, where $\gamma(G)$ is the domination number of $G$, and this is nearly sharp: for $t, m, r \in \mathbb{N}$ with $t \leq m$, there is a graph $G$ with domination number $t$ such that $\sigma(G, m, r)>t(r / m-1)$. Also, there are chordal graphs (and bipartite graphs) for given $r$ and $m$ such that $\sigma(G, m, r)=r-m+1$. If $G$ is the $d$-dimensional hypercube $Q_{d}$ with $d \geq r$, then $\sigma(G, 2, r)=r-1$; in general, if $d \geq r \geq m \geq 3$, then $\sigma\left(Q_{d}, m, r\right)>r-39 m$. 
They also consider $r$-large complete $k$-partite graphs, where " $r$-large" means that each partite set has size at least $2 r$. When $k \geq m$ and $G$ is such a graph,

$$
\frac{k \cdot k\lfloor r / k\rfloor}{(k-1) m+1}-k \leq \sigma(G, m, r) \leq\left\lceil\frac{k}{k-1} \frac{r}{m}\right\rceil+k .
$$

When $G$ is a large bipartite graph, they show that $\sigma(G, 2, r)=7 r / 10$ and $\sigma(G, 3, r)=3 r / 2$ (within additive constants) and that in general

$$
\frac{\lfloor r / 2\rfloor}{\lceil m / 3\rceil}-2 \leq \sigma(G, m, r) \leq\left(1+\frac{1}{\sqrt{3}}\right) \frac{r}{m}+1 .
$$

The upper bound coefficient on $\frac{r}{m}$ is about 1.58; the lower one is about 1.5 when $m$ is large and $r>m$; they conjecture that the lower one is the true coefficient, at least when $3 \mid \mathrm{m}$.

The rules for movement in the game of Revolutionaries and Spies are the same as in the classical game of Cops-and-Robber. Imagine that there is one revolutionary (the robber) and that the spies (cops) take positions first. The difference is in the winning condition: the cops win if a cop ever moves to the same location as the robber. The cop number [4] is the minimum number of cops needed to win the game. The graphs with cop number 1 have been characterized [3]. Many variations of the game have been studied, with more than 50 papers on the subject.

\section{Trees and cycles}

If $r / m<|V(G)|$, then the spies lose whenever $s<\lfloor r / m\rfloor$. Hence the first chance for the spies is when $s=\lfloor r / m\rfloor$. We prove that this suffices when $G$ is a tree, yielding $\sigma(G, m, r)=\lfloor r / m\rfloor$. Since the spies always win when $s \geq|V(G)|$, the statement of our first theorem remains true regardless of the relationship between $r / m$ and $|V(G)|$. Nevertheless, to avoid trivial statements, we henceforth assume always that $r / m \leq|V(G)|$.

Theorem 1. If $G$ is a tree and $s \geq\lfloor r / m\rfloor$, then the spies win $\operatorname{RS}(G, m, r, s)$.

Proof. It suffices to show that the spies win when $s=\lfloor r / m\rfloor<|V(G)|$. Choose a root $z \in V(G)$. The parent of a non-root vertex $v$, denoted $v^{+}$, is its neighbor on the path from $v$ to $z$. The other neighbors of $v$ are its children; let $C(v)$ be the set of children of $v$. The descendants of $v$ are all the vertices (including $v$ ) whose path to $z$ contains $v$; let $D(v)$ be the set of descendants of $v$. 
For each vertex $v$, let $r(v)$ and $s(v)$ denote the number of revolutionaries and spies on $v$ at the current time, respectively, and let $w(v)=\sum_{u \in D(v)} r(u)$. The spies maintain the following invariant giving the number of spies on each vertex at the end of any round:

$$
s(v)=\left\lfloor\frac{w(v)}{m}\right\rfloor-\sum_{u \in C(v)}\left\lfloor\frac{w(u)}{m}\right\rfloor \quad \text { for } v \in V(G) .
$$

Since $\sum_{u \in C(v)} w(u)=w(v)-r(v)$, the formula is always nonnegative. Also, if $r(v) \geq m$, then $s(v) \geq\left\lfloor\frac{w(v)}{m}\right\rfloor-\left\lfloor\frac{w(v)-r(v)}{m}\right\rfloor \geq 1$. Hence (1) guarantees that every meeting is guarded.

To show that the spies can establish (1) after the first round, it suffices that all the formulas sum to $\lfloor r / m\rfloor$. More generally, summing over the descendants of any vertex $v$,

$$
\sum_{u \in D(v)} s(u)=\left\lfloor\frac{w(v)}{m}\right\rfloor
$$

since $\lfloor w(u) / m\rfloor$ occurs positively in the term for $u$ and negatively in the term for $u^{+}$, except that $\lfloor w(v) / m\rfloor$ occurs only positively. When $v=z$, the total is $\lfloor r / m\rfloor$, since $w(z)=r$.

To show that the spies can maintain (1), let $r^{\prime}(v)$ denote the new number of revolutionaries at $v$ after the revolutionaries move, and let $w^{\prime}(v)=$ $\sum_{u \in D(v)} r^{\prime}(v)$. The spies move to achieve the new values required by (1), starting from the leaves; we will produce $s^{\prime}(v)$ at $v$ after adjusting at all the children (and lower descendants) of $v$.

We process siblings simultaneously. That is, having updated all children of $C(v)$, we adjust at all of $C(v)$ simultaneously. In doing this, excess spies move to $v$, and needed spies come from $v$; no changes are made involving children of $C(v)$. Similarly, fixing $C\left(v^{+}\right)$later includes an exchange between $v$ and $v^{+}$but does not disturb the spies on $C(v)$. After successfully updating $C(v)$ for all $v$, the root vertex $z$ will have exactly $s^{\prime}(z)$ spies, since always $\sum s^{\prime}(v)=\sum s(v)=\lfloor r / m\rfloor$.

We can now process $C(v)$. Let $D^{*}(u)=D(u)-\{u\}$ for all $u$. For $u \in$ $C(v)$, all vertices in $D^{*}(u)$ have been adjusted. Spies previously on $D^{*}(u)$ remained in $D(u)$, and those now on $D^{*}(u)$ came from $D(u)$. Hence $u$ now has $\sum_{t \in D(u)} s(t)-\sum_{t \in D^{*}(u)} s^{\prime}(t)$ spies. Let

$$
\partial(u)=s^{\prime}(u)-\sum_{t \in D(u)} s(t)+\sum_{t \in D^{*}(u)} s^{\prime}(t)=\left\lfloor\frac{w^{\prime}(u)}{m}\right\rfloor-\left\lfloor\frac{w(u)}{m}\right\rfloor .
$$


As defined, changing by $\partial(u)$ achieves $s^{\prime}(u)$ spies at $u$; the second equality uses (2). When $\partial(u)$ is positive, $\partial(u)$ spies move from $v$ to $u$; when it is negative, $u$ sends $-\partial(u)$ spies to $v$.

Let $C^{+}=\left\{u \in C(v): w^{\prime}(u)>w(u)\right\}$ and $C^{-}=C(v)-C^{+}$. Note that both $C^{+}$and $C^{-}$may contain vertices for whom the adjustment from or to $v$ is 0 . To avoid making spies take two steps to reach $C^{+}$, we must ensure $\sum_{u \in C^{+}} \partial(u) \leq s(v)$. To avoid forcing spies from $C^{-}$to take a second step, we must ensure $\sum_{u \in C^{-}} \partial(u) \leq s^{\prime}(v)$.

For the first inequality, note that $\sum_{u \in C^{+}}\left[w^{\prime}(u)-w(u)\right] \leq r(v)$, since revolutionaries who entered subtrees rooted at $C(v)$ on this round were previously at $v$. Thus

$$
\begin{aligned}
\sum_{u \in C^{+}}\left\lfloor\frac{w^{\prime}(u)}{m}\right\rfloor & \leq\left\lfloor\sum_{u \in C^{+}} \frac{w^{\prime}(u)}{m}\right\rfloor \\
& \leq\left\lfloor\frac{r(v)+\sum_{u \in C^{+}} w(u)}{m}\right\rfloor=\left\lfloor\frac{w(v)-\sum_{u \in C^{-}} w(u)}{m}\right\rfloor \\
& \leq\left\lfloor\frac{w(v)}{m}\right\rfloor-\sum_{u \in C^{-}}\left\lfloor\frac{w(u)}{m}\right\rfloor=s(v)+\sum_{u \in C^{+}}\left\lfloor\frac{w(u)}{m}\right\rfloor .
\end{aligned}
$$

By (3), this yields the desired inequality. For the second inequality, $\sum_{u \in C^{-}}\left[w(u)-w^{\prime}(u)\right] \leq r^{\prime}(v)$, since revolutionaries who left subtrees rooted at $C^{-}$on this round are now at $v$. Thus

$$
\begin{aligned}
\sum_{u \in C^{-}}\left\lfloor\frac{w(u)}{m}\right\rfloor & \leq\left\lfloor\sum_{u \in C^{-}} \frac{w(u)}{m}\right\rfloor \\
& \leq\left\lfloor\frac{r^{\prime}(v)+\sum_{u \in C^{-}} w^{\prime}(u)}{m}\right\rfloor=\left\lfloor\frac{w^{\prime}(v)-\sum_{u \in C^{+}} w^{\prime}(u)}{m}\right\rfloor \\
& \leq\left\lfloor\frac{w^{\prime}(v)}{m}\right\rfloor-\sum_{u \in C^{+}}\left\lfloor\frac{w^{\prime}(u)}{m}\right\rfloor=s^{\prime}(v)+\sum_{u \in C^{-}}\left\lfloor\frac{w^{\prime}(u)}{m}\right\rfloor .
\end{aligned}
$$

Again (3) yields the desired inequality.

These inequalities ensure that the adjustments restore (1) by legal moves. The adjustment to $C(v)$ is allowed, after which $v$ has $s(v)-\sum_{u \in C(v)} \partial(u)$ spies. Using (1) and (3),

$$
s(v)-\sum_{u \in C(v)} \partial(u)=s^{\prime}(v)-\left\lfloor\frac{w^{\prime}(v)}{m}\right\rfloor+\left\lfloor\frac{w(v)}{m}\right\rfloor=s^{\prime}(v)-\partial(v) .
$$


Thus, when $C\left(v^{+}\right)$is later processed, the adjustment needed at $v$ is exactly what we have said will be made. Furthermore, spies moving to $v$ from $C^{-}$do not come from below $C^{-}$and do not continue on to $v^{+}$. The argument for the latter applies also to the former. If $\partial(v)<0$, requiring $-\partial(v)$ spies to move to $v^{+}$, then the adjustment at $C(v)$ left more than $s^{\prime}(v)$ spies at $v$. However, since $\sum_{u \in C^{-}} \partial(u) \leq s^{\prime}(v)$, the extra spies beyond $s^{\prime}(v)$ were among the $s(v)$ spies on $v$ at the beginning of the round, so they can move to $v^{+}$.

Corollary 2. If $G$ is a forest and $s \geq\lfloor r / m\rfloor$, then the spies win $\operatorname{RS}(G, m$, $r, s)$.

We next show that $\lceil r / m\rceil$ spies suffice to win on a cycle.

Lemma 3. If $G$ is a cycle, then $\sigma(G, m, r) \leq\lceil r / m\rceil$. Equality holds when $m \mid r$ and $r / m \leq|V(G)|$.

Proof. Extra revolutionaries cannot make the game easier for the spies, so we may assume that $r=s m$. Given the initial locations of revolutionaries, index the revolutionaries in order from 0 to $s m-1$ around the cycle. Place spies on the vertices occupied by the revolutionaries whose index is a multiple of $m$ (this may put more than one spy on a vertex).

Because revolutionaries are identical, we may assume that the revolutionaries always remain indexed in order around the cycle as they move (equivalently, if revolutionaries switch positions, then they trade indices). Since indices move along at most one edge in each round, the $i$ th spy can continue to follow the revolutionary with index $\mathrm{im}$. Thus after every round, any vertex occupied by at least $m$ revolutionaries is guarded by at least one spy.

When the cycle is short enough, the threshold for a spy win improves to the trivial lower bound $\lfloor r / m\rfloor$. For longer cycles, the strategy for the revolutionaries to defeat $\lfloor r / m\rfloor$ spies may take many rounds to produce an unguarded meeting. Note that when $r \mid m$, the upper bound from Lemma 3 coincides with the trivial lower bound to yield $\sigma\left(C_{n}, m, r\right)=r / m$ when $n \geq r / m$.

Theorem 4. If $G$ is a cycle of length $\ell$, and $m \nmid r$ with $r / m \leq \ell$, then $\sigma(G, m, r)=\lfloor r / m\rfloor$ if and only if $\ell \leq\lfloor r / m\rfloor+2$ or $r<m$.

Proof. Let $s=\lfloor r / m\rfloor$. When $r<m$, no meetings can be formed, so no spies are needed. When $\ell \leq s+2$, the spies can guard all initial meetings and leave at most two vertices unguarded. After a move by the revolutionaries, at least two vertices $u$ and $v$ fail to host a meeting. The spies can move to leave only $u$ and $v$ unguarded, by shifting one step along paths from $u$ and $v$ to the previously unguarded vertices. 
For the converse, consider $\ell \geq s+3$. It suffices to show that the revolutionaries win when $r=s m+1$. They will first distract one spy $S$, arranging for $S$ to guard a vertex occupied by at most one revolutionary. They then win by making $s$ meetings on the remaining path, guarded by at most $s-1$ spies.

The revolutionaries move so that no more than $m$ of them ever occupy one vertex. Subject to this, they start with any initial distribution. The spies take initial positions, and the revolutionaries designate one spy as $S$. To reduce what $S$ guards, on each round the revolutionaries guarded by $S$ move away, half in one direction and half in the other (rounded to integers). The revolutionaries that were on the neighboring vertices move farther away, but still each vertex has at most $m$ revolutionaries; this is possible since $\ell \geq s+3$. No matter how $S$ moves, the number of revolutionaries guarded by $S$ is at most half (rounded up) of what it was before. After at most $\left\lceil\log _{2} m\right\rceil$ rounds, $S$ guards at most one revolutionary.

Now the revolutionaries shorten the path containing the other revolutionaries by moving those nearest to $S$ away from $S$, maintaining that each vertex has at most $m$ revolutionaries (again possible since $\ell \geq s+3$ ). They continue until the path consists of $s$ consecutive vertices with meetings, which cannot all be guarded by the $s-1$ spies other than $S$.

\section{Unicyclic graphs}

A connected unicyclic graph has a cycle and trees attached to it. The cycle and trees interact, since revolutionaries may move on and off the cycle. The spies must respond appropriately.

Theorem 5. If $G$ is a unicyclic graph, then $\sigma(G, m, r) \leq\lceil r / m\rceil$.

Proof. It suffices to show that the spies win $\operatorname{RS}(G, m, r, s)$ when $s=\lceil r / m\rceil \leq$ $|V(G)|$. If there are $r_{i}$ revolutionaries in a component $G_{i}$ of $G$, and $G_{i}$ is a tree, then only $\left\lfloor r_{i} / m\right\rfloor$ spies are needed in $G_{i}$. Since at most one component contains a cycle, and $\lfloor a\rfloor+\lceil b\rceil \leq\lceil a+b\rceil$ for all $a, b \in \mathbb{R}$, we may assume that $G$ is connected and contains a cycle $C$. View $G-V(C)$ as disjoint trees rooted at vertices neighboring $C$. In order to use the strategies of Theorem 1 and Lemma 3, a spy must be available when needed to move from $C$ to a tree (or vice versa).

Say that the current state satisfies the cycle condition if the number of revolutionaries on $C$ is $m$ times the number of spies on $C$ and there is a spy guarding every $m$ th revolutionary as described in the proof of Lemma 3. The key fact needed is that adding one spy and $m$ revolutionaries to any vertex of $C$ (or removing them) preserves the cycle condition. 
As in Lemma 3, we may assume $m \mid r$. We may also assume that all revolutionaries start on the cycle. To play against another initial position, the spies imagine an initial position on the cycle and follow their winning strategy as the revolutionaries move to the actual start. With all revolutionaries initially on the cycle, the cycle condition holds at the start.

The attached trees are the components of $G-V(C)$. The root of an attached tree $T$ is the vertex $z$ adjacent to a vertex of $C$. The neighbor of $z$ in $V(C)$, denoted $z^{*}$, is the mate of $T$. As revolutionaries disperse to or return from the attached trees, maintaining the cycle condition requires keeping a buffer of "fake" revolutionaries for each tree at its mate on $C$ (a vertex may be the mate of many trees).

When a revolutionary moves from $C$ to an attached tree $T$, it moves to the root $z$ of $T$ from the mate $z^{*}$ on $C$. Until $m$ revolutionaries move to $T$, no spy needs to follow, since $m-1$ revolutionaries cannot make a meeting on $T$. For each revolutionary that moves from $z^{*}$ to $z$, we add a fake revolutionary at $z^{*}$; this maintains the cycle condition. The fake revolutionaries are markers maintained by the spies and do not move.

When $m$ actual revolutionaries have moved to $T$, a spy is needed. Before the final move of $j$ revolutionaries from $z^{*}$ to $z$, there were also $m-j$ fake revolutionaries at $z^{*}$, so by the cycle condition there was a spy at $z^{*}$. This spy moves to $z$ following the $j$ revolutionaries, and $m-j$ fake revolutionaries disappear from $z^{*}$. This preserves the cycle condition.

In the strategy on $T$ given in Theorem 1, when revolutionaries are added at the root to increase the number of spies needed, the vertex needing the extra spy is the root. Hence the arrival of the spy at $z$ from $z^{*}$ permits the spy strategy on $T$ to operate locally. Similarly, when revolutionaries leave $T$ to reduce the number of spies needed, they leave the root. The number of spies computed for other vertices of $T$ does not change, so the location of the extra spy is $z$. It can return to $z^{*}$ and reestablish the appropriate number of fake revolutionaries.

As spies follow revolutionaries onto and off the cycle, the fake revolutionaries enable the spies to maintain the cycle condition, and the strategies for spies on the cycle and on the attached trees can operate independently as previously given.

By Theorem 5, $\sigma(G, m, r) \in\{\lfloor r / m\rfloor,\lceil r / m\rceil\}$ when $G$ is unicyclic and $|V(G)| \geq r / m$. Theorem 6 determines which is the answer. The role of vertices outside the cycle is shown by the disjoint union of $C_{5}$ and $P_{2}$. Three spies defeat seven revolutionaries on $C_{5}$ when $m=2$, by Theorem 4 . However, four revolutionaries can sit on $P_{2}$ forever, occupying two spies, and the remaining three revolutionaries defeat the remaining spy on $C_{5}$. Adding an 
edge to join the two components does not affect the revolutionaries' strategy; it does not matter whether the graph is connected.

Theorem 6. Let $G$ be a unicyclic graph having a cycle $C$ of length $l$ and exactly $t$ vertices not on $C$. If $|V(G)| \geq r / m$, then $\sigma(G, m, r) \in\{\lfloor r / m\rfloor,\lceil r / m\rceil\}$, equalling $\lfloor r / m\rfloor$ when $m \nmid r$ if and only if $\ell \leq \max (\lfloor r / m\rfloor-t+2,3)$ or $r<m$.

Proof. By Theorem 5, $\sigma(G, m, r) \leq\lceil r / m\rceil$, whether or not $G$ is connected. Assume $m \nmid r$, and let $s=\lfloor r / m\rfloor \leq|V(G)|$. It suffices to show that the revolutionaries win $\operatorname{RS}(G, m, r, s)$ if $\ell \geq \max \{s-t+3,4\}$, and the spies win $\operatorname{RS}(G, m, r, s)$ if $\ell \leq \max \{s-t+2,3\}$.

When $\ell \geq \max \{s-t+3,4\}$, the revolutionaries first make meetings at $k$ vertices outside $C$, where $k=\min \{t, s-1\}$. These meetings must be guarded by $k$ spies. These $k m$ revolutionaries (and thus also $k$ spies) will not move (it does not matter whether their component contains $C$ ). On $C$, the remaining $r-k m$ revolutionaries play against $s-k$ spies. Since $r / m>s$, also $(r-k m) / m>s-k$. Since $s-k=\max \{s-t, 1\}$, we have $\ell \geq s-k+3$. By Theorem 4, the remaining revolutionaries win on the cycle.

Now suppose $\ell \leq \max \{s-t+2,3\}$. If $G$ has a component $H$ with $\hat{t}$ vertices and $\hat{r}$ revolutionaries outside the component containing $C$, then $H$ needs only $\min \{\hat{t},\lfloor\hat{r} / m\rfloor\}$ spies. On $G-V(H)$, since $\lfloor a\rfloor+\lfloor b\rfloor \leq\lfloor a+b\rfloor$, the specified conditions hold for the remaining spies to win. Hence to consider a minimal counterexample we may assume that $G$ is connected. As in Theorem 4, we may also assume that all revolutionaries start on $C$.

Case 1. $s>t$. In this case, $\ell \leq s-t+2$. Since $|V(G)|>\lfloor r / m\rfloor=s$, in fact $\ell \in\{s-t+1, s-t+2\}$. Each attached tree $T$ reserves $|V(T)|$ spies, located initially at the mate of $T$. These "tree spies" mostly remain at the mate of $T$ except to move into $T$ as needed to play the spy strategy on $T$ from Theorem 5. When $r_{T}$ revolutionaries are in $T$, exactly $\left\lfloor r_{T} / m\right\rfloor$ tree spies will have followed them. By Theorem 1, the revolutionaries never make an unguarded meeting outside $C$.

The $s-t$ unreserved spies always occupy distinct vertices of $C$; call them "cycle spies". To draw $t^{\prime}$ tree spies off $C$, at least $m t^{\prime}$ revolutionaries must have left $C$. Since $s=\lfloor r / m\rfloor$, fewer than $m\left(s-t^{\prime}+1\right)$ revolutionaries remain on $C$. Hence at most $s-t^{\prime}$ meetings on $C$ need to be guarded. Since $\ell \leq s-t+2$, we have seen in Theorem 4 that the $s-t$ cycle spies can move to guard any desired set of $s-t$ vertices on $C$.

If at least $\ell-(s-t)$ vertices of $C$ retain tree spies, then the cycle spies can guard the remaining vertices of $C$. If no vertices of $C$ retain tree spies, then $t^{\prime}=t$. In this case fewer than $m(s-t+1)$ revolutionaries are on $C$, they make at most $s-t$ meetings, and the cycle spies can guard those meetings. 
Hence we may assume that $\ell=s-t+2$ and that all tree spies on $C$ are at one vertex, $v$. The cycle spies can guard all the other meetings on $C$ unless there are $s-t+1$ such meetings. If there is also a meeting at $v$, then there are at least $m(s-t+2)$ revolutionaries on $C$. Hence $m(s-t+2)<m\left(s-t^{\prime}+1\right)$, which yields $t^{\prime}<t-1$. Thus at least two tree spies remain at $v$, and one of them can move to guard a meeting at a neighbor $u$ of $v$ on $C$. If there is no meeting at $v$, then again a tree spy from $v$ can guard a meeting at $u$.

This is the only way a tree spy leaves its reserved subgraph; when the condition ends the spy moves back. It cannot be pulled into $T$ (two steps) at the same time, because having $m(s-t+1)$ revolutionaries on $V(C)-\{v\}$ leaves fewer than $m t$ revolutionaries in the union of the trees and $v$ : not enough to pull the last tree spy back into its tree.

Case 2. $s \leq t$. In this case, $\ell=3$, and the vertices of $C$ are pairwise adjacent. Deleting the edges of $C$ leaves three disjoint trees, rooted at the vertices of $C$. As usual, the spies may assume that the revolutionaries all start on $V(C)$. Always, an initial position can be defended by $\lfloor r / m\rfloor$ spies, and in this case they are all on the cycle.

At a given time, let $r_{i}$ be the number of revolutionaries on the component $T_{i}$ of $G-E(C)$ (rooted at $v_{i} \in V(C)$ ), for $i \in\{1,2,3\}$. The spies maintain that at the end of each round there are at least $\left\lfloor r_{i} / m\right\rfloor$ spies on tree $T_{i}$, arranged according to the strategy of Theorem 1, with any extra spies located at the root $v_{i}$. Since each vertex appears in some $T_{i}$, ability to maintain this invariant completes the proof. The invariant holds after the initial placement, since revolutionaries appear only at the roots.

After the revolutionaries move, the spies update their position on each $T_{i}$ via the strategy in the proof of Theorem 1 . The update starts from the leaves and works toward the root. The revolutionaries now at non-root vertices of $T_{i}$ were in $T_{i}$ at the end of the previous round, since revolutionaries enter or leave $T_{i}$ only via edges of $C$. As shown in Theorem 1 , the updates to all vertices except the root can be completed using spies that were in $T_{i}$ before the round, since the invariant held at that time. Also, spies who moved to $v_{i}$ during this process are covering revolutionaries who came there from $T_{i}-v_{i}$.

Let $s_{i}=\left\lfloor r_{i} / m\right\rfloor$. If not enough revolutionaries arrive at $v_{i}$ from the rest of $C$ on this round to push the number of revolutionaries on $T_{i}$ up to $m s_{i}+m$, then the invariant already holds on $T_{i}$. However, if $T_{i}$ now contains at least $m s_{i}+m k$ revolutionaries (for some positive $\mathrm{k}$ ), then the number of revolutionaries remaining in the other trees is at most $r-m\left(s_{i}+k\right)$, so the number of spies needed on those trees is at most $s-s_{i}-k$. That is, $k$ spies are freed to move to $v_{i}$. Furthermore, since the new revolutionaries 
in $T_{i}$ came from the other roots and the tree strategy was followed using $\left\lfloor r_{j} / m\right\rfloor$ spies on each $T_{j}$, the freed spies were at the other roots and are now available to move to $v_{i}$. Doing so restores the desired invariant.

The technique of Case 2 above does not work in Case 1, since the revolutionaries can make the vertex on $C$ needing extra spies be far from the vertex with freed spies.

We have now determined the winner for every game $\mathrm{RS}(G, m, r, s)$ such that $G$ is unicyclic, and we have provided a constructive strategy for the winner in each case.

\section{References}

[1] J. V. Butterfield, D. W. Cranston, G. J. Puleo, D. B. West, and R. Zamani, Revolutionaries and spies: Spy-good and spy-bad graphs. Theoretical Comp. Sci., to appear.

[2] D. Howard and C. D. Smyth, Revolutionaries and spies on grid-like graphs. Discrete Math., to appear.

[3] R. Nowakowski, and P. Winkler, Vertex-to-vertex pursuit in a graph. Discrete Math. 43 (1983), 235-239. MR0685631

[4] A. Quilliot, A short note about pursuit games played on a graph with a given genus. J. Combin. Theory Ser. B 38 (1985), 89-92. MR0782627

Daniel W. Cranston

Virginia Commonwealth University

USA

E-mail address: dcranston@vcu.edu

ClifFord D. SMYTH

University of North Carolina

Greensboro

USA

E-mail address: cdsmyth@uncg.edu

Douglas B. West

ZheJiANG Normal University

China

UNIVERSITY OF ILLINOIS

USA

E-mail address: west@math.uiuc.edu

Received September 27, 2011 